\title{
Article \\ Some Conditions on Trans-Sasakian Manifolds to Be Homothetic to Sasakian Manifolds
}

\author{
Sharief Deshmukh ${ }^{1}\left(\mathbb{0}\right.$, Amira Ishan ${ }^{2}\left(\mathbb{D}\right.$, Olga Belova $^{3, *}{ }^{\circledR}$ and Suha B. Al-Shaikh ${ }^{4}(\mathbb{C}$ \\ 1 Department of Mathematics, College of Science, King Saud University, P.O. Box 2455, \\ Riyadh 11451, Saudi Arabia; shariefd@ksu.edu.sa \\ 2 Department of Mathematics, College of Science, Taif University, P.O. Box 11099, Taif 21944, Saudi Arabia; \\ a.ishan@tu.edu.sa \\ 3 Institute of Physical and Mathematical Sciences and IT, Immanuel Kant Baltic Federal University, \\ A. Nevsky Str. 14, 236016 Kaliningrad, Russia \\ 4 Information Technology Department, Arab Open University, P.O. Box 84901, Hittin 11681, Saudi Arabia; \\ s.alshaikh@arabou.edu.sa \\ * Correspondence: olgaobelova@mail.ru; Tel.: +7-921-610-5949
}

Citation: Deshmukh, S.; Ishan, A.; Belova, O.; Al-Shaikh, S.B. Some Conditions on Trans-Sasakian Manifolds to Be Homothetic to Sasakian Manifolds. Mathematics 2021, 9, 1887. https://doi.org/ 10.3390/math9161887

Academic Editor: Marian Ioan Munteanu

Received: 4 July 2021

Accepted: 6 August 2021

Published: 8 August 2021

Publisher's Note: MDPI stays neutral with regard to jurisdictional claims in published maps and institutional affiliations.

Copyright: (C) 2021 by the authors. Licensee MDPI, Basel, Switzerland. This article is an open access article distributed under the terms and conditions of the Creative Commons Attribution (CC BY) license (https:// creativecommons.org/licenses/by/ $4.0 /)$.

\begin{abstract}
In this paper, we study 3-dimensional compact and connected trans-Sasakian manifolds and find necessary and sufficient conditions under which these manifolds are homothetic to Sasakian manifolds. First, four results in this paper deal with finding necessary and sufficient conditions on a compact and connected trans-Sasakian manifold to be homothetic to a compact and connected Sasakian manifold, and the fifth result deals with finding necessary and sufficient condition on a connected trans-Sasakian manifold to be homothetic to a connected Sasakian manifold. Finally, we find necessary and sufficient conditions on a compact and simply connected trans-Sasakian manifold to be homothetic to a compact and simply connected Einstein Sasakian manifold.
\end{abstract}

Keywords: trans-Sasakian manifolds; Sasakian manifolds; Einstein Sasakian manifolds; scalar curvature

\section{Introduction}

It is well known that the product $\bar{M}=M \times R$ of a $(2 n+1)$-dimensional almost contact metric manifold $(M, F, \mathbf{t}, u, g)$ (cf. Reference [1]) has an almost complex structure $J$, which with product metric $\bar{g}$ makes $(\bar{M}, \bar{g})$ an almost Hermitian manifold. The geometry of the almost contact metric manifold $(M, F, \mathbf{t}, u, g)$ depends on the geometry of the almost Hermitian manifold $(\bar{M}, J, \bar{g})$ and gives several structures on $M$ like a Sasakian structure, a quasi-Sasakian structure, and others (cf. References [1-3]). There are sixteen different types of structures on the almost Hermitian manifold $(\bar{M}, J, \bar{g})$ (cf. Reference [4]), and the structure in the class $\mathcal{W}_{4}$ on $(\bar{M}, J, g)$ gives a structure $(F, \mathbf{t}, u, g, \alpha, \beta)$ on $M$ known as transSasakian structure (cf. Reference [5]), which generalizes a Sasakian structure, a Kenmotsu structure, and a cosymplectic structure on a contact metric manifold (cf. References [2,3]), where $\alpha, \beta$ are smooth functions defined on $M$. Here, the class $\mathcal{W}_{4}$ should not be confused with Stiefel-Whitney characteristic class, but it is one of the sixteen classes specified by different combinations of covariant derivatives of the almost complex structure $J$ on the almost Hermitian manifold.

A trans-Sasakian manifold $(M, F, \mathbf{t}, u, g, \alpha, \beta)$ is called a trans-Sasakian manifold of type $(\alpha, \beta)$ and trans-Sasakian manifolds of type $(0,0),(\alpha, 0)$ and $(0, \beta)$ are called cosymplectic, $\alpha$-Sasakian and $\beta$-Kenmotsu manifolds, respectively. In Reference [6], Marrero proved that a trans-Sasakian manifold of dimension greater than or equal to five is either a cosymplectic manifold, a $\alpha$-Sasakian manifold, or a $\beta$-Kenmotsu manifold; therefore, after this result, there is an emphasis in studying geometry of 3-dimensional trans-Sasakian manifolds. We shall abbreviate a 3-dimensional trans-Sasakian manifold $(M, F, \mathbf{t}, u, g)$ by a TRS-manifold. 
An interesting question is to seek conditions under which a closed (compact without boundary) TRS-manifold is homothetic to a Sasakian manifold. The geometry of TRSmanifold is important because of Thurston's conjecture (cf. Reference [7]), now known as Geometrization-Conjecture, which gave eight geometries on a 3-dimensional manifold, namely Spherical geometry $\mathbf{S}^{3}$, Euclidean geometry $\mathbf{E}^{3}$, Hyperbolic geometry $\mathbf{H}^{3}$, the geometry of $\mathbf{S}^{2} \times \mathbf{R}$, the geometry of $\mathbf{H}^{2} \times \mathbf{R}$, the geometry of universal cover of $\mathbf{S L}(\mathbf{2}, \mathbf{R})$, the Nil geometry, and the Sol geometry (for details on this topic, see Reference [8]). In addition, we know that 3-dimensional Sasakian manifolds are in abundance, for example, the unit sphere $\mathbf{S}^{3}$, the Euclidean space $\mathbf{E}^{3}$, the unit tangent bundle $\mathbf{T}_{1} \mathbf{S}^{2}$ of the sphere $\mathbf{S}^{2}$, the special unitary group $\mathbf{S U}(\mathbf{2})$, the Heisenberg group $\mathcal{H}^{3}$, and the special linear group $\operatorname{SL}(2, \mathbf{R})$ (cf. Reference [9]). Thus, the geometry of TRS-manifolds, in matching them with Thurston's eight geometries on 3-dimensional closed Riemannian manifolds, becomes more interesting, and, as we see, many in the list of Thurston's geometries are included in the list of Sasakian manifolds.

In References [10-19], the authors studied compact TRS-manifolds with some restrictions on the smooth functions $\alpha, \beta$ and the vector field $\mathbf{t}$ appearing in their definition for getting conditions under which a TRS-manifold is homothetic to a Sasakian manifold. It is known that a compact simply connected TRS-manifold satisfying Poisson equations $\Delta \alpha=\beta, \Delta \alpha=\alpha^{2} \beta$, respectively, gives a necessary and sufficient condition for it to be homothetic to a Sasakian manifold (cf. Reference [13]).

In addition, in References [19-21], interesting results on the geometry of TRS-manifolds are obtained, where the authors (W. Wang, X. Liu, Y. Wang, Y. Zhao) considered other aspects in Thurston's eight geometries. In Reference [13], a question was asked whether the function $\beta$ on a compact TRS-manifold satisfying the differential equation grad $\beta=\mathfrak{t}(\beta) \mathbf{t}$ necessitates the TRS-manifold to be homothetic to a Sasakian manifold. It is shown that this question has negative answer (cf. Reference [21]). However, with additional restrictions, such as positivity of sectional curvatures, and certain differential inequality satisfied by the function, $\beta$ gives an affirmative answer to this question (cf. Theorem 3.5, [15]).

Owing to Thurston's geometrization conjecture, geometry of TRS-manifolds (being 3-dimensional Riemannian manifolds) have become an important subject. Moreover, Sasakian geometry picks up many important geometries in Thurston's eight geometries; hence, the question of finding conditions under which a TRS-manifold is homothetic to a Sasakian manifold has considerable importance. In Section 3 of this paper, the first four results deal with finding necessary and sufficient conditions on a compact connected TRS-manifold $(M, F, \mathbf{t}, u, g, \alpha, \beta)$ to be homothetic to a compact and connected Sasakian manifold, and the fifth deals with finding necessary and sufficient conditions on a connected TRS-manifold $(M, F, \mathbf{t}, u, g, \alpha, \beta)$ to be homothetic to a connected Sasakian manifold.

In the first result, we consider a compact connected TRS-manifold $(M, F, \mathbf{t}, u, g, \alpha, \beta)$ of constant scalar curvature $\tau$ satisfying the inequality $\tau \leq 6\left(\alpha^{2}+\beta^{2}\right)$ and the Ricci operator $T$ satisfying $T(\mathbf{t})=\frac{\tau}{3} \mathbf{t}$, and we give necessary and sufficient conditions for $M$ to be homothetic to a compact and connected Sasakian manifold (see Theorem 2). In the second result, we show that a compact and connected TRS-manifold $(M, F, \mathbf{t}, u, g, \alpha, \beta)$ with Ricci curvature $S(\mathbf{t}, \mathbf{t})$ a non-zero constant and satisfying $S(\mathbf{t}, \mathbf{t}) \leq 2\left(\alpha^{2}+\beta^{2}\right)$ give necessary and sufficient conditions for $M$ to be homothetic to a compact and connected Sasakian manifold (see Theorem 3). Similarly, in the third result, we show that conditions $S(\mathbf{t}, \mathbf{t}) \neq 0$ and $F(\operatorname{grad} \alpha)=\operatorname{grad} \beta$ on a compact and connected TRS-manifold $(M, F, \mathbf{t}, u, g, \alpha, \beta)$ are necessary and sufficient for $M$ to be homothetic to a compact and connected Sasakian manifold (see Theorem 4). In addition, the fourth result deals with conditions $\alpha(p) \neq 0$ for a point $p \in M$ and $F(\operatorname{grad} \beta)=-\operatorname{grad} \alpha$ on a compact and connected TRS-manifold $(M, F, \mathbf{t}, u, g, \alpha, \beta)$ to reach a similar conclusion (see Theorem 5). Finally, in the fifth result, we show that compactness could be dropped with the conditions $\alpha(p) \neq 0$ for a point $p \in M$ and $\|$ grad $\alpha \|^{2}=4 \alpha^{2} \beta^{2}$ on a connected TRS-manifold $(M, F, \mathbf{t}, u, g, \alpha, \beta)$ to reach a similar conclusion (see Theorem 6). 
Among Sasakian manifolds, Einstein Sasakian manifolds play an important role because of their elegant geometry, as well as their important applications in theoretical physics (see the excellent monograph in Reference [9]). In the last section of this paper, we find necessary and sufficient conditions on a compact and simply connected TRSmanifold $(M, F, \mathbf{t}, u, g, \alpha, \beta)$ to be homothetic to a compact simply connected Einstein Sasakian manifold (see Theorem 7).

\section{Preliminaries}

Let $(M, F, \mathbf{t}, u, g)$ be a 3-dimensional almost contact metric manifold, where $F$ is a $(1,1)$-tensor field, $\mathbf{t}$ a unit vector field, and $u$ a smooth 1 -form dual to $\mathbf{t}$ with respect to the Riemannian metric $g$, satisfying

$$
F^{2}=-I+u \otimes \mathbf{t}, F(\mathbf{t})=0, u \circ F=0, g(F U, F V)=g(U, V)-u(U) u(V),
$$

$U, V \in \mathfrak{X}(M)$, where $\mathfrak{X}(M)$ is the Lie algebra of smooth vector fields on $M$ (cf. Reference [1]). If there are smooth functions $\alpha, \beta$ on an almost contact metric manifold $(M, F, \mathbf{t}, u, g)$ satisfying

$$
(\nabla F)(U, V)=\alpha(g(U, V) \mathbf{t}-u(V) U)+\beta(g(F U, V) \mathbf{t}-u(V) F U),
$$

then $(M, F, \mathbf{t}, u, g, \alpha, \beta)$ is said to be a trans-Sasakian manifold, where $(\nabla F)(U, V)=$ $\nabla_{U} F V-F\left(\nabla_{U} V\right), \quad U, V \in \mathfrak{X}(M)$, and $\nabla$ is the Levi-Civita connection with respect to the metric $g$ (cf. References $[7,10-15,20]$ ). We shall abbreviate the 3-dimensional trans-Sasakian manifold $(M, F, \mathbf{t}, u, g, \alpha, \beta)$ by TRS-manifold. Using Equations (1) and (2), we get

$$
\nabla_{U} \mathbf{t}=-\alpha F(U)+\beta(U-u(U) \mathbf{t}), \quad U \in \mathfrak{X}(M) .
$$

Let $S$ be the Ricci tensor of a Riemannian manifold $(M, g)$. Then, the Ricci operator $T$ is defined by $S(U, V)=g(T U, V), U, V \in \mathfrak{X}(M)$. On a TRS-manifold, we have the following:

$$
\begin{gathered}
\mathbf{t}(\alpha)=-2 \alpha \beta, \\
T(\mathbf{t})=F(\operatorname{grad} \alpha)-\operatorname{grad} \beta+2\left(\alpha^{2}-\beta^{2}\right) \mathbf{t}-\mathbf{t}(\beta) \mathbf{t} .
\end{gathered}
$$

Note that Equation (3) implies

$$
\operatorname{div} \mathbf{t}=2 \beta,
$$

and, using this equation, together with Equation (4), we have

$$
\operatorname{div}\left(\alpha^{m} \mathbf{t}\right)=m \alpha^{m-1} \mathbf{t}(\alpha)+\alpha^{m} \operatorname{div} \mathbf{t}=-2 m \alpha^{m} \beta+2 \alpha^{m} \beta=-2(m-1) \alpha^{m} \beta .
$$

Thus, on a compact TRS-manifold, using Equation (6) and the above equation, we have

$$
\int_{M} \beta=0, \quad \int_{M} \alpha^{m} \beta=0 \text { for } m \neq 1 .
$$

Now, we state the following result of Okumura.

Theorem 1 (Reference [18]). Let $(M, g)$ be a Riemannian manifold. If $M$ admits a Killing vector field $\mathbf{t}$ of constant length satisfying

$$
\alpha^{2}\left(\nabla_{U} \nabla_{V} \mathbf{t}-\nabla_{\nabla_{U} V} \mathbf{t}\right)=g(V, \mathbf{t}) U-g(U, V) \mathbf{t}
$$

for non-zero constant $\alpha$ and any vector fields $U$ and $V$, then $M$ is homothetic to a Sasakian manifold. 
Given two Riemannian manifolds $\left(M_{1}, g_{1}\right),\left(M_{2}, g_{2}\right)$, a diffeomorphism $f: M_{1} \rightarrow M_{2}$ is said to be a conformal transformation if the pullback $f^{*}\left(g_{2}\right)$ of the metric $g_{2}$ satisfies

$$
f^{*}\left(g_{2}\right)=e^{\sigma} g_{1},
$$

where $\sigma$ is a smooth function on $M_{1}$. If the function $\sigma$ is a constant, then the diffeomorphism $f$ is said to be a homothety, and, in this situation, the Riemannian manifold $\left(M_{1}, g_{1}\right)$ is said to be homothetic to the Riemannian manifold $\left(M_{2}, g_{2}\right)$. Thus, Theorem 1 gives a condition under which a Riemannian manifold $(M, g)$ is homothetic to a Sasakian manifold.

For a smooth function $f$ on a Riemannian manifold $(M, g)$, the Hessian operator $A_{f}$ of $f$ is defined by

$$
A_{f}(U)=\nabla_{\text {ugradf }}, \quad U \in \mathfrak{X}(M),
$$

and the Laplace operator $\Delta$ is defined by $\Delta f=\operatorname{div}(\operatorname{grad} f)$, and it satisfies

$$
\Delta f=\operatorname{tr} A_{f} .
$$

\section{TRS-Manifolds Homothetic to Sasakian Manifolds}

In this section, we find necessary and sufficient conditions on a TRS-manifold $(M, F, \mathbf{t}, u$, $g, \alpha, \beta)$ to be homothetic to a Sasakian manifold.

Theorem 2. A compact and connected TRS-manifold $(M, F, \mathbf{t}, u, g, \alpha, \beta)$ with constant scalar curvature $\tau$ satisfying

$$
\tau \leq 6\left(\alpha^{2}+\beta^{2}\right)
$$

is homothetic to a compact and connected Sasakian manifold of constant scalar curvature if and only if the Ricci operator T satisfies

$$
T(\mathbf{t})=\frac{\boldsymbol{\tau}}{3} \mathbf{t} .
$$

Proof. Suppose $T(\mathbf{t})=\frac{\tau}{3} \mathbf{t}$ holds, then, using Equation (5), we have

$$
F(\operatorname{grad} \alpha)-\operatorname{grad} \beta+2\left(\alpha^{2}-\beta^{2}-\frac{\tau}{6}-\frac{1}{2} \mathbf{t}(\beta)\right) \mathbf{t}=0 .
$$

Taking the inner product in the above equation with $\mathbf{t}$, we get

$$
\mathbf{t}(\beta)=\alpha^{2}-\beta^{2}-\frac{\tau}{6} .
$$

Using Equation (6), we have $\operatorname{div}(\beta \mathbf{t})=\mathbf{t}(\beta)+2 \beta^{2}$, and, by (8), we have

$$
\operatorname{div}(\beta \mathbf{t})-2 \beta^{2}=\alpha^{2}-\beta^{2}-\frac{\tau}{6} .
$$

Integrating the above equation, we conclude

$$
\int_{M}\left(\alpha^{2}+\beta^{2}-\frac{\tau}{6}\right)=0
$$

Using the inequality in the statement, we get

$$
\alpha^{2}+\beta^{2}=\frac{\tau}{6} .
$$

Since, $\tau$ is a constant, we get $M$ is homothetic to a Sasakian manifold of constant scalar curvature (cf. Theorem 3.1, in Reference [14]). The converse is trivial. 
Theorem 3. A compact and connected TRS-manifold $(M, F, \mathbf{t}, u, g, \alpha, \beta)$ with Ricci curvature $S(\mathbf{t}, \mathbf{t})$ a non-zero constant is homothetic to a compact and connected Sasakian manifold if and only if

$$
S(\mathbf{t}, \mathbf{t}) \leq 2\left(\alpha^{2}+\beta^{2}\right)
$$

Proof. Using Equation (5), we have

$$
S(\mathbf{t}, \mathbf{t})=2\left(\alpha^{2}-\beta^{2}-\mathbf{t}(\beta)\right)
$$

Now, using $\operatorname{div}(\beta \mathbf{t})=\mathbf{t}(\beta))+2 \beta^{2}$ in the above equation, we get

$$
S(\mathbf{t}, \mathbf{t})-2\left(\alpha^{2}-\beta^{2}\right)+2 \operatorname{div}(\beta \mathbf{t})-4 \beta^{2}=0 .
$$

Integrating the above equation, we have

$$
\int_{M}\left(S(\mathbf{t}, \mathbf{t})-2\left(\alpha^{2}+\beta^{2}\right)\right)=0 .
$$

Using condition in the statement, we conclude

$$
S(\mathbf{t}, \mathbf{t})=2\left(\alpha^{2}+\beta^{2}\right)
$$

Combining Equations (9) and (10), we arrive at $\mathbf{t}(\beta)=-2 \beta^{2}$, i.e., $3 \beta^{2} \mathbf{t}(\beta)=-6 \beta^{4}$ or $\mathbf{t}\left(\beta^{3}\right)=-6 \beta^{4}$. Thus, using Equation (6), we have

$$
\operatorname{div}\left(\beta^{3} \mathbf{t}\right)=-6 \beta^{4}+2 \beta^{4}=-4 \beta^{4}
$$

Integrating the above equation, we conclude that $\beta=0$. Consequently, Equation (9) implies $S(\mathbf{t}, \mathbf{t})=2 \alpha^{2}$, and, as the Ricci curvature $S(\mathbf{t}, \mathbf{t})$ is non-zero constant, we conclude $\alpha$ is a non-zero constant. The Equation (3) now takes the form

$$
\nabla_{U} \mathbf{t}=-\alpha F(U), \quad U \in \mathfrak{X}(M),
$$

and we get

$$
\left(£_{\mathbf{t}} g\right)(U, V)=-\alpha g(F(U), V)-\alpha g(F(V), U)=0, \quad U, V \in \mathfrak{U}(M),
$$

i.e., the unit vector field $\mathbf{t}$ is Killing. Moreover, using Equation (11), we get

$$
\alpha^{-2}\left(\nabla_{U} \nabla_{V} \mathbf{t}-\nabla_{\nabla_{U} V} \mathbf{t}\right)=g(V, \mathbf{t}) U-g(U, V) \mathbf{t},
$$

where $\alpha$ is a non-zero constant. Hence, by Theorem 1, we conclude that $M$ is homothetic to a Sasakian manifold. The converse is trivial as for a Sasakian manifold $S(\mathbf{t}, \mathbf{t})=2$.

Theorem 4. A compact and connected TRS-manifold $(M, F, \mathbf{t}, u, g, \alpha, \beta)$ with the Ricci curvature $S(\mathbf{t}, \mathbf{t}) \neq 0$ is homothetic to a compact and connected Sasakian manifold, if and only if, $F($ grad $\alpha)=$ $\operatorname{grad} \beta$.

Proof. Suppose $F(\operatorname{grad} \alpha)=\operatorname{grad} \beta$. Then, we have $\Delta \beta=\operatorname{div} F(\operatorname{grad} \alpha)$ and

$$
\operatorname{div} F(\operatorname{grad} \alpha)=\sum_{i=1}^{3} g\left(\nabla_{e_{i}} F(\operatorname{grad} \alpha), e_{i}\right)=\sum_{i=1}^{3} g\left((\nabla F)\left(e_{i}, \operatorname{grad} \alpha\right)+F\left(A_{\alpha} e_{i}\right), e_{i}\right),
$$


where $\left\{e_{1}, e_{2}, e_{3}\right\}$ is a local orthonormal frame on $M$, and $A_{\alpha}$ is the Hessian operator of $\alpha$. Using $\operatorname{Tr} F A_{\alpha}=0$ and Equations (2) and (4), we get

$$
\operatorname{div} F(\operatorname{grad} \alpha)=-2 \alpha \mathbf{t}(\alpha)=4 \alpha^{2} \beta .
$$

Thus, we have $\Delta \beta=4 \alpha^{2} \beta$, i.e.,

$$
\beta \Delta \beta=4 \alpha^{2} \beta^{2} .
$$

Integrating by parts the above equation gives

$$
-\int_{M}\|\operatorname{grad} \beta\|^{2}=4 \int_{M} \alpha^{2} \beta^{2} .
$$

The above integral implies

$$
\int_{M}\|\operatorname{grad} \beta\|^{2} \leq 0,
$$

i.e., $\|\operatorname{grad} \beta\|^{2}=0$, and it gives $\beta$ is a constant. Now, using Equation (7), we conclude $\beta=0$. Then, we have $F(\operatorname{grad} \alpha)=0$, and, operating $F$ on this equation while using Equation (1), we get

$$
\operatorname{grad} \alpha=\mathbf{t}(\alpha) \mathbf{t} .
$$

In order to accomplish the result, we intend to use Theorem 3 by showing that $S(\mathbf{t}, \mathbf{t}) \leq 2\left(\alpha^{2}+\beta^{2}\right)$. However, $\beta=0$ in Equation (4) implies $\mathbf{t}(\alpha)=0$; therefore, the above equation implies $\alpha$ is a constant. Moreover, using Equation (5), we get $S(\mathbf{t}, \mathbf{t})=2 \alpha^{2}$, and, using the condition in the statement, we get $\alpha$ is a non-zero constant. Thus, as in Theorem 3 , we conclude that $M$ is homothetic to a Sasakian manifold. The converse is trivial.

Theorem 5. A compact and connected TRS-manifold $(M, F, \mathbf{t}, u, g, \alpha, \beta)$ with $\alpha(p) \neq 0$ for a point $p \in M$, is homothetic to a compact and connected Sasakian manifold, if and only if, $F(\operatorname{grad} \beta)=-\operatorname{grad} \alpha$.

Proof. Suppose $F(\operatorname{grad} \beta)=-\operatorname{grad} \alpha$ holds. Then, we have

$$
F(\operatorname{grad} \alpha)-\operatorname{grad} \beta=-\mathbf{t}(\beta) \mathbf{t},
$$

and, inserting above equation in Equation (5), we arrive at

$$
T(\mathbf{t})=2\left(\alpha^{2}-\beta^{2}-\mathbf{t}(\beta)\right) \mathbf{t} .
$$

In addition, using similar steps as in Theorem 4 , we compute $\operatorname{div} F(\operatorname{grad} \beta)=-2 \alpha \mathbf{t}(\beta)$. Thus, we have $\Delta \alpha=2 \alpha \mathbf{t}(\beta)$, i.e., $\alpha \Delta \alpha=2 \alpha^{2} \mathbf{t}(\beta)$. Integrating this equation, we get

$$
-\int_{M}\|\operatorname{grad} \alpha\|^{2}=2 \int_{M} \alpha^{2} \mathbf{t}(\beta) .
$$

Now, we observe that

$$
\operatorname{div}\left(\beta\left(\alpha^{2} \mathbf{t}\right)\right)=\alpha^{2} \mathbf{t}(\beta)+\beta \operatorname{div}\left(\alpha^{2} \mathbf{t}\right)=\alpha^{2} \mathbf{t}(\beta)+2 \alpha \beta \mathbf{t}(\alpha)+\alpha^{2} \beta \operatorname{div} \mathbf{t},
$$

and, using Equations (4) and (6) in the above equation, we get

$$
\operatorname{div}\left(\beta\left(\alpha^{2} \mathbf{t}\right)\right)=\alpha^{2} \mathbf{t}(\beta)-2 \alpha^{2} \beta^{2},
$$


and, by Equation (12), we conclude

$$
-\int_{M}\|\operatorname{grad} \alpha\|^{2}=4 \int_{M} \alpha^{2} \beta^{2}
$$

which implies grad $\alpha=0$. Hence, $\alpha$ is a constant, which through the condition in the statement implies $\alpha$ is a non-zero constant. Moreover, we have $\mathbf{t}(\alpha)=0$, and, in view of Equation (4), we have $\beta=0$. Thus, as in Theorem 3, we conclude that $M$ is homothetic to a Sasakian manifold. The converse is trivial.

We observe that Equation (4) implies $g(\mathbf{t}, g r a d \alpha)^{2}=4 \alpha^{2} \beta^{2}$, and, with $\mathbf{t}$ being a unit vector field, it implies $4 \alpha^{2} \beta^{2} \leq\|\operatorname{grad} \alpha\|^{2}$. Naturally, one feels prompted to ask what happens in case of the equality. Interestingly, the answer is the TRS-manifold which, in this case, is homothetic to a Sasakian manifold without imposition of compactness, as seen in the following result.

Theorem 6. A connected TRS-manifold $(M, F, \mathbf{t}, u, g, \alpha, \beta)$ with $\alpha(p) \neq 0$ for a point $p \in M$ is homothetic to a connected Sasakian manifold, if and only if, $\|$ grad $\alpha \|^{2}=4 \alpha^{2} \beta^{2}$.

Proof. Suppose that $\|g r a d \alpha\|^{2}=4 \alpha^{2} \beta^{2}$ holds. Then, using Equation (4), we have

$$
\begin{aligned}
\|\operatorname{grad} \alpha+2 \alpha \beta \mathbf{t}\|^{2} & =\|\operatorname{grad} \alpha\|^{2}+4 \alpha^{2} \beta^{2}+4 \alpha \beta \mathbf{t}(\alpha) \\
& =\|\operatorname{grad} \alpha\|^{2}-4 \alpha^{2} \beta^{2}=0,
\end{aligned}
$$

i.e.,

$$
\operatorname{grad} \alpha=-2 \alpha \beta \mathbf{t}
$$

Taking covariant derivative in the above equation with respect to $U \in \mathfrak{X}(M)$ and using Equation (3), we arrive at

$$
A_{\alpha} U=2 \alpha^{2} \beta F(U)-2 \alpha \beta^{2} U-2\left(U(\alpha \beta)-2 \alpha \beta^{2} u(U)\right) \mathbf{t} .
$$

Now, using symmetry of the operator $A_{\alpha}$, the above equation implies

$$
4 \alpha^{2} \beta g(F(U), V)-2 U(\alpha \beta) u(V)+2 V(\alpha \beta) u(U)=0, \quad U, V \in \mathfrak{X}(M),
$$

and $U=\mathbf{t}$ in the above equation yields

$$
\mathbf{t}(\alpha \beta) u(V)=V(\alpha \beta), \quad V \in \mathfrak{X}(M),
$$

i.e.,

$$
\operatorname{grad}(\alpha \beta)=\mathbf{t}(\alpha \beta) \mathbf{t}
$$

Using Equations (4) and (13) in the above equation, we arrive at

$$
\alpha(\operatorname{grad} \beta-\mathbf{t}(\beta) \mathbf{t})=0 .
$$

Note that, as in the statement $\alpha \neq 0$ and, accordingly, on connected $M$, the above equation implies

$$
\operatorname{grad} \beta=\mathbf{t}(\beta) \mathbf{t}
$$

Using Equation (14), we get

$$
2 \alpha^{2} \beta F(U)=U(\alpha \beta) \mathbf{t}-u(U) \operatorname{grad}(\alpha \beta), \quad U \in \mathfrak{X}(M) .
$$


The above equation, together with Equations (4), (13), (15), and (16) in the above equation, we conclude

$$
2 \alpha^{2} \beta F(U)=0, \quad U \in \mathfrak{X}(M),
$$

i.e., $\alpha^{2} \beta=0$. Since $\alpha \neq 0$, we get $\beta=0$. Thus, Equation (13) implies $\alpha$ is a non-zero constant, and, using Equations (2) and (3), we get

$$
\alpha^{-2}\left(\nabla_{U} \nabla_{V} \mathbf{t}-\nabla_{\nabla_{U} V} \mathbf{t}\right)=g(V, \mathbf{t}) U-g(U, V) \mathbf{t},
$$

and this proves $M$ is homothetic to a connected Sasakian manifold (see Theorem 1). The converse is trivial.

\section{TRS-Manifolds Homothetic to Einstein Sasakian Manifolds}

Recall that, among Sasakian manifolds, Einstein Sasakian manifolds have elegant geometry (cf. Reference [9]), as well as play a vital role in physics (see References [22,23] for details). In this section, we prove the following:

Theorem 7. A compact and simply connected TRS-manifold $(M, F, \mathbf{t}, u, g, \alpha, \beta)$ with $T(\mathbf{t})=$ $2\left(\alpha^{2}+\beta^{2}\right) \mathbf{t}$, is homothetic to a compact and simply connected Sasakian manifold if and only if

$$
(\nabla T)(U, \mathbf{t})=(\nabla T)(\mathbf{t}, U), \quad U \in \mathfrak{X}(M) .
$$

Proof. Suppose $T(\mathbf{t})=2\left(\alpha^{2}+\beta^{2}\right) \mathbf{t}$ holds. Then, using Equation (5) gives

$$
F(\operatorname{grad} \alpha)=\operatorname{grad} \beta+4 \beta^{2} \mathbf{t}+\mathbf{t}(\beta) \mathbf{t},
$$

and, taking the inner product with $\mathbf{t}$ in the above equation, we get $\mathbf{t}(\beta)=-2 \beta^{2}$. Thus, we have $3 \beta^{2} \mathbf{t}(\beta)=-6 \beta^{4}$, i.e., $\mathbf{t}\left(\beta^{3}\right)=-6 \beta^{4}$, and, using Equation (6), we conclude

$$
\operatorname{div}\left(\beta^{3} \mathbf{t}\right)=-4 \beta^{4}
$$

Integrating the above equation confirms $\beta=0$. Then, Equations (4) and (17) imply $\mathbf{t}(\alpha)=0$ and $F(\operatorname{grad} \alpha)=0$, and, operating $F$ on the second equation, we get grad $\alpha=0$, i.e., $\alpha$ is a constant. Now, $M$ being simply connected, we claim that $\alpha$ is a non-zero constant. For, if $\alpha=0$, then, by Equation (3), the vector field $\mathbf{t}$ is parallel; therefore, $u$ is closed and has to be exact, and there exists a smooth function $\varphi$ such that $u=d \varphi$. Thus, $\mathbf{t}=\operatorname{grad} \varphi$, and, as $M$ is compact, there exists a point $x \in M$ such that $(\operatorname{grad} \varphi)(x)=0$, i.e., $\mathbf{t}(x)=0$, which is a contradiction to the fact that $\mathbf{t}$ is a unit vector field. Hence, constant $\alpha \neq 0$ and, with $\beta=0$, Equations (2) imply the Lie derivative

$$
£_{t} g=0
$$

This proves that $\mathbf{t}$ is a Killing vector field, and the flow of $\mathbf{t}$ consists of isometries of $M$, and, as such, we have

$$
\left(£_{t} T\right)(U)=0, \quad U \in \mathfrak{X}(M),
$$

and the above equation in view of Equation (3) implies

$$
(\nabla T)(\mathbf{t}, U)=\alpha T(F U)-\alpha F(T U), \quad U \in \mathfrak{X}(M) .
$$

Using the condition in the statement, we have

$$
(\nabla T)(U, \mathbf{t})=\alpha T(F U)-\alpha F(T U), \quad U \in \mathfrak{X}(M) .
$$


Now, with $\beta=0$ and $\alpha$ a constant, we have $T(\mathbf{t})=2 \alpha^{2} \mathbf{t}$, and, taking covariant derivative in this equation while using Equation (3), we have

$$
(\nabla T)(U, \mathbf{t})+T(-\alpha F U)=-2 \alpha^{3} F U, \quad U \in \mathfrak{X}(M) .
$$

Combining Equations (18) and (19) with constant $\alpha \neq 0$, we get

$$
F(T U)=2 \alpha^{2} F U, \quad U \in \mathfrak{X}(M),
$$

and, operating $F$ on the above equation, and keeping in mind the equation $T(\mathbf{t})=2 \alpha^{2} \mathbf{t}$, we have

$$
T U=2 \alpha^{2} U, \quad U \in \mathfrak{X}(M),
$$

i.e., $M$ is an Einstein manifold. Now, with $\alpha$, a non-zero constant, and $\beta=0$, using Equations (2) and (3), we conclude

$$
\alpha^{-2}\left(\nabla_{U} \nabla_{V} \mathbf{t}-\nabla_{\nabla_{U} V} \mathbf{t}\right)=g(V, \mathbf{t}) U-g(U, V) \mathbf{t} .
$$

Hence, by virtue of Theorem 1 , we get $M$ is homothetic to a compact simply connected Einstein Sasakian manifold. The converse is trivial.

Author Contributions: Conceptualization and methodology, S.D., A.I., S.B.A.-S.; formal analysis, O.B.; writing—original draft preparation, S.D., O.B., A.I., S.B.A.-S.; writing—review and editing, S.D. and O.B.; supervision, S.D.; project administration. All authors have read and agreed to the published version of the manuscript.

Funding: This research received no external funding.

Institutional Review Board Statement: Not applicable.

Informed Consent Statement: Not applicable.

Data Availability Statement: Not applicable.

Acknowledgments: This work is supported by Taif University Researchers Supporting Project number (TURSP-2020/223), Taif University, Taif, Saudi Arabia.

Conflicts of Interest: The authors declare no conflict of interest.

\section{References}

1. Blair, D.E. Contact Manifolds in Riemannian Geometry; Lecture Notes in Mathematics; Springer: Berlin, Germany; New York, NY, USA, 1976; pp. 395-407.

2. Blair, D.E.; Oubiña, J.A. Conformal and related changes of metric on the product of two almost contact metric manifolds. Publ. Mater. 1990, 34, 199-207. [CrossRef]

3. Fujimoto, A.; Muto, H. On cosymplectic manifolds. Tensor 1974, 28, 43-52.

4. Gray, A.; Hervella, L.M. The sixteen classes of almost Hermitian manifolds and their linear invariants. Ann. Mater. Pura Appl. 1980, 123, 35-58. [CrossRef]

5. Oubiña, J.A. New classes of almost contact metric structures. Publ. Math. Debrecen 1985, 32, 187-193.

6. Marrero, J.C. The local structure of trans-Sasakian manifolds. Ann. Mater. Pura Appl. 1992, 162, 77-86. [CrossRef]

7. Thurston, W.P. Three dimensional manifolds, Kleinian groups and hyperbolic geometries. Bull. Am. Math. Soc. 1982, 6, 357-381. [CrossRef]

8. Bessieres, L.; Besson, G.; Boileau, M.; Maillot, S.; Porti, J. Geometrisation of 3-manifolds. In EMS Tracts in Mathematics; European Mathematical Society: Zurich, Switzerland, 2010; Volume 13.

9. Boyer, C.; Galicki, K. Sasakian Geometry; Oxford Mathematical Monographs; Oxford University Press: Oxford, UK, 2008.

10. De, U.C.; Sarkar, A. On three-Dimensional trans-Sasakian manifolds. Extracta Math. 2008, 23, 265-277. [CrossRef]

11. Deshmukh, S.; Tripathi, M.M. A note on trans-Sasakian manifolds. Math. Slov. 2013, 63, 1361-1370. [CrossRef]

12. Deshmukh, S.; De, U.C.; Al-Solamy, F. Trans-Sasakian manifolds homothetic to Sasakian manifolds. Publ. Math. Debr. 2016, 88, 439-448. [CrossRef]

13. Deshmukh, S. Trans-Sasakian manifolds homothetic to Sasakian manifolds. Mediterr. J. Math. 2016, 13, 2951-2958. [CrossRef]

14. Deshmukh, S.; Alsolamy, F. A note on compact Trans-Sasakian manifolds. Mediterr. J. Math. 2016, 13, 2099-2104. [CrossRef]

15. Deshmukh, S.; De, U.C. A note on Trans-Sasakian manifolds. arXiv 2018, arxiv:1203.0860.

16. Ma, R.; Pei, D. Reeb flow invariant *-Ricci operators on trans-Sasakian three-Manifolds. Math. Slov. 2021, 71, 749-756. [CrossRef] 
17. Obata, M. Certain conditions for a Riemannian manifold to be isometric with a sphere. J. Math. Soc. Jpn. 1962, 14, 333-340. [CrossRef]

18. Okumura, M. Certain almost contact hypersurfaces in Kaehlerian manifolds of constant holomorphic sectional curvatures. Tohoku Math. J. 1964, 16, 270-284. [CrossRef]

19. Zhao, Y. Some characterizations of property of trans-Sasakian 3-Manifolds. Math. Slov. 2021, 71, 383-390. [CrossRef]

20. Wang, W.; Liu, X. Ricci tensors on trans-Sasakian 3-manifolds. Filomat 2018, 32, 4365-4374. [CrossRef]

21. Wang, Y.; Wang, W. A remark on trans-Sasakian 3-manifolds. Rev. Union Mater. Argent. 2019, 60, 257-264. [CrossRef]

22. Maldacena, J.M. The large N limit of superconformal field theories and supergravity. Adv. Theor. Math. Phys. 1998, 2, 231-252. [CrossRef]

23. Slesar, V.; Visinescu, M.; Vîlcu, G.E. Toric data, Killing forms and complete integrability of geodesics in Sasaki-Einstein spaces $Y^{p, q}$. Ann. Phys. 2015, 361, 548-562. [CrossRef] 